\title{
Hydrogenation of bilayer graphene and the formation of bilayer graphane from first principles
}

\author{
O. Leenaerts, ${ }^{1, *}$ B. Partoens, ${ }^{1, \dagger}$ and F. M. Peeters ${ }^{1, \ddagger}$ \\ ${ }^{1}$ Departement Fysica, Universiteit Antwerpen, Groenenborgerlaan 171, B-2020 Antwerpen, Belgium \\ (Received 23 September 2009; revised manuscript received 9 November 2009; published 15 December 2009)
}

\begin{abstract}
We performed $a b$ initio density-functional theory calculations to investigate the process of hydrogenation of a bilayer of graphene. 50\% hydrogen coverage is possible in case that the hydrogen atoms are allowed to adsorb on both sides of the bilayer. In this case interlayer chemical bonding occurs which stabilizes the structure. At maximum coverage, a bilayer of graphane is formed which has properties that are similar to those of a single layer of graphane.
\end{abstract}

DOI: $10.1103 /$ PhysRevB.80.245422

PACS number(s): 68.43.Bc, 68.43.Fg, 81.05.Uw, 73.21.Ac

\section{INTRODUCTION}

In search for new materials for future electronics, graphene ${ }^{1}$ has recently been suggested as a suitable candidate because of its exceptional properties such as its essentially two-dimensional form and high crystal quality, electron mobility, and robustness. ${ }^{2-4}$ Some major obstacles which have to be dealt with before graphene can make its promises come true are the absence of a band gap in the electronic spectrum of intrinsic graphene and the Klein paradox as a consequence of the Dirac-type nature of the charge carriers. These obstacles can be more easily overcome in a bilayer of graphene because it is possible to induce a tunable band gap here by applying a gate voltage ${ }^{5,6}$ or through chemical modification of the surface. The most studied way to chemically modify a graphene surface is undoubtly hydrogenation. This is probably due to the simplicity of these systems and additionally the possible application of graphene systems for hydrogen storage. ${ }^{7}$ This last application has resulted in a lot of research on the interaction of molecular and atomic hydrogen with graphitic materials. In this research it was found that molecular hydrogen leads to physisorption whereas atomic hydrogen can form chemical bonds with the carbon atoms. In case of only a few layers of graphene, it was found that hydrogenating a single layer of graphene is easier than hydrogenating a bilayer. ${ }^{8,9}$

In this paper we investigate the hydrogenation of a bilayer of graphene. We examine the maximum coverage of $\mathrm{H}$ atoms on the bilayer and the stability of these hydrogenated systems. We will show that when both sides of the bilayer are exposed to atomic hydrogen and when adsorption takes place on one of the two crystal sublattices (on each side), the weak van der Waals (vdW) bonding is replaced by strong chemical bonds between the two layers which stabilize the system and lead to a new material. Chemical bonding between graphene layers has been investigated before in the context of graphite fluorination ${ }^{10}$ or irradiated graphite in which case point defects (e.g., vacancies and interstitial atoms) can induce interlayer bonding. ${ }^{11}$

We find that the coverage of hydrogen atoms on bilayer graphene can reach a maximum of 50 at. \% and in this case a bilayer analog of graphane $e^{8,12}$ is formed. The geometrical bond lengths and angles of this bilayer of graphane are between those of graphane and diamond but the electronic properties are similar to those of single-layer graphane.
This paper is organized as follows: first we describe the computational details of our ab initio calculations in which special attention is paid to the choice of exchange-correlation functional. Then, we examine the possibility and the stability of interlayer chemical bonds in bilayer graphene. To conclude, we investigate the geometrical and electronic properties of bilayer graphane and compare them to single layer graphane and diamond.

\section{COMPUTATIONAL DETAILS}

All our calculations were done within the densityfunctional theory formalism as implemented in the ABINIT simulation code. ${ }^{13}$ We made use of the projector augmentedwave method ${ }^{14}$ and a plane-wave basis set with a cutoff energy of 20 hartree. Different supercells, ranging from $2 \times 2$ to $4 \times 4$, were used for simulation of the different systems. The sampling of the Brillouin zone was done for these different supercells with the equivalent of a $12 \times 12 \times 1$ Monkhorst-Pack $k$-point grid $^{15}$ for a single graphene unit cell. Spin polarization was included where necessary. ${ }^{16}$ For most calculations we made of the local-density approximation (LDA) for the exchange-correlation functional which is believed to give a better description of the interlayer binding in graphite than the generalized gradient approximation (GGA). ${ }^{17}$ The fact that LDA gives a better description of the interaction between graphene layers is rather accidental and results from a cancellation of errors. We tested our LDA simulation procedure for graphite and diamond and compare the results with GGA and experimental data. The results of

TABLE I. Properties of graphite and diamond: the unit cell lengths $(a, c)$ are given in $\AA$ and the interlayer binding energy $E_{B}$ in meV.

\begin{tabular}{lcccc}
\hline \hline & & LDA & GGA & Experiment \\
\hline Graphite & $a$ & 2.445 & 2.465 & $2.46^{\mathrm{a}}$ \\
& $c$ & 6.712 & 8.64 & $6.67^{\mathrm{b}}$ \\
& $E_{B}$ & 26 & $\sim 2$ & $61 \pm 5^{\mathrm{b}}$ \\
Diamond & $a$ & 3.529 & 3.578 & $3.57^{\mathrm{c}}$ \\
\hline
\end{tabular}

${ }^{\text {aReference } 18 .}$

${ }^{\mathrm{b}}$ Reference 19.

${ }^{\mathrm{c}}$ Reference 20. 


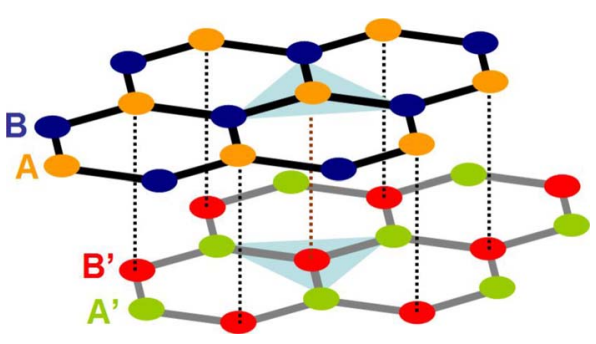

FIG. 1. (Color online) Bilayer of $A B$ stacked graphene with the four different sublattices indicated by different shades (colors). The $A$ and $B^{\prime}$ sublattices are situated on top of each other as illustrated by the dotted lines. The carbon atoms at the corners of the light triangles indicate the six neighbors to be hydrogenated.

these test cases, given in Table I, are consistent with earlier LDA (and GGA) calculations ${ }^{17,21}$ but, as is typical with LDA, the bond lengths are a little underestimated in comparison with the experimental results. The interlayer binding energy $\Delta E$ in graphite is only half the experimental value. This is not a big issue for the work presented here as the energies involved in chemical bonding, which are of interest here, are one to two orders of magnitude larger. The GGA results give a more accurate description of the (in-plane) lattice constant $(a)$ in graphite and diamond but the interplane distance $(c / 2)$ and binding energy in graphite are much better reflected by the LDA results. So, as stated above, LDA is to be preferred in this work.

\section{RESULTS}

The main focus of this paper is on the formation of chemical bonds between the two layers of graphene as a consequence of hydrogen adsorption. Therefore we first investigate whether this is possible, in principle, or not. If any chemical bond between the two layers is possible, it is more likely to occur between two carbon atoms belonging to different layers that are closest to each other, i.e., atoms of the $\mathrm{A}$ and $\mathrm{B}^{\prime}$ sublattices as defined in Fig. 1. For the formation of a chemical bond between these atoms, it is necessary they change their hybridization from $s p^{2}$ to $s p^{3}$ because they have to be able to form four bonds now.

We can take two neighboring atoms of different layers and bring them closer together to form a chemical bond but it is obvious that this cannot result in a stable system because the deformation of the two layers costs much more energy than can be gained by forming a new bond: when the two atoms form a new bond they move out of the graphene planes and force their neighbors to change their hybridization from $s p^{2}$ because the three bonds formed by these neighboring atoms are not lying in a plane. It is reasonable to suggest that hydrogenation of the neighboring atoms (i.e., atoms of the $\mathrm{B}$ and $\mathrm{A}^{\prime}$ sublattices) can result in a stable system because this makes sure they already have the right hybridization. For this, hydrogen atoms must be able to chemisorb on pure bilayer graphene which was recently shown theoretically to be possible. ${ }^{22,23}$

In an $A B$ stacked bilayer of graphene the $\mathrm{A}$ and $\mathrm{B}$ (or $\mathrm{A}^{\prime}$ and $\mathrm{B}^{\prime}$ ) sublattices are no longer completely equivalent which results in a different adsorption energy for a hydrogen atom on these two sublattices: adsorption on the $\mathrm{B}$ (or $\mathrm{A}^{\prime}$ ) sublattice is more favorable by $0.03 \mathrm{eV}$ as compared to adsorption on the A (or $\mathrm{B}^{\prime}$ ) sublattice. Although this difference is small, it might be important for interlayer bonding because the carbon atoms that should be hydrogenated belong to the more favorable sublattice. The two carbon atoms involved in the interlayer bonding each have three neighbors that need to be hydrogenated to get the right hybridization (see Fig. 1). However it may be sufficient to hydrogenate some of these neighbors to make the interlayer bond already stable.

To investigate this we use a $2 \times 2$ supercell of bilayer graphene containing 16 carbon atoms (eight per layer) and start to increase gradually the hydrogenation of the six neighbors (three of the $\mathrm{B}$ and three of the $\mathrm{A}^{\prime}$ sublattices) of the two carbon atoms between which we are looking for a possible interlayer chemical bond. We see from Table II that there is a jump in the minimal interlayer C-C distance $\left(d_{\min }\right)$ after the adsorption of three hydrogen atoms, two on one layer, and one on the other. This indicates the formation of an interlayer chemical bond as illustrated in Fig. 2(a). Adsorption of hydrogen atoms on a single sublattice of one side

TABLE II. Interlayer chemical bond formation in a $2 \times 2$ supercell. The binding energy per $\mathrm{H}$ atom $\left(E_{b}\right)$ and the average $\left(d_{\text {avg }}\right)$ and minimum distance $\left(d_{\text {min }}\right)$ between the $\mathrm{C}$ atoms of the two layers are shown for different numbers of adsorbed hydrogen atoms and different configurations. Energies are given in $\mathrm{eV}$ and distances in $\AA$.

\begin{tabular}{cccccc}
\hline \hline H atoms & Configuration & $E_{b}$ & $d_{\text {avg }}$ & $d_{\text {min }}$ & Chemical bond \\
\hline 1 & $B$ & -1.084 & 3.274 & 3.231 & No \\
2 & $B B$ & Not stable & & & \\
2 & $B A^{\prime}$ & -1.266 & 2.965 & 2.853 & No \\
3 & $B B B$ & Not stable & & & Yes \\
3 & $B B A^{\prime}$ & -1.326 & 2.564 & 1.660 & Yes \\
4 & $B B B A^{\prime}$ & -1.426 & 2.440 & 1.591 & Yes \\
4 & $B B A^{\prime} A^{\prime}$ & -2.018 & 2.347 & 1.600 & Yes \\
5 & $B B B A^{\prime} A^{\prime}$ & -2.036 & 2.278 & 1.574 & Yes \\
6 & $B B B A^{\prime} A^{\prime} A^{\prime}$ & -2.295 & 2.025 & 1.550 & \\
\hline \hline
\end{tabular}


a)

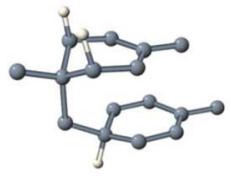

b)

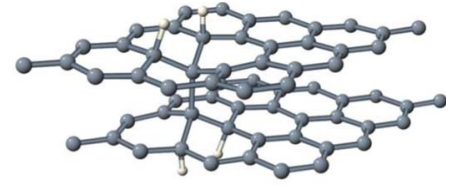

c)

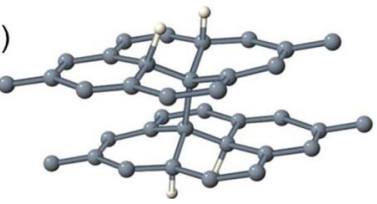

d)

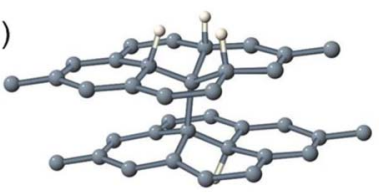

FIG. 2. (Color online) Formation of a chemical bond between the two layers in bilayer graphene in (a) a $2 \times 2$, (b) a $4 \times 4$, and [(c) and (d)] a $3 \times 3$ supercell. The light colored atoms are $\mathrm{H}$ and the others $\mathrm{C}$.

does not result in any stable structure in a $2 \times 2$ supercell. Notice that the average interlayer distance $\left(d_{\text {avg }}\right)$ becomes smaller when more carbon atoms are hydrogenated. This means that the carbon atoms of the different layers, that do not participate in interlayer bond formation, start repelling each other more and more. For lower concentrations of $\mathrm{H}$ atoms, i.e., larger supercells, there are more repelling carbon atoms so it is likely that the interlayer bond needs to be stronger. We checked this in a $3 \times 3$ and a $4 \times 4$ supercell and show the results in Table III: in a $3 \times 3$ (36 C atoms) supercell one needs two hydrogenated neighbors on each side [see Fig. 2(c)], so four in total or three on one side and one on the other side of bilayer graphene [Fig. 2(d)]. In a $4 \times 4$ supercell (64 $\mathrm{C}$ atoms) both sides need at least two hydrogenated neighbors [Fig. 2(b)]. For still lower concentrations (thus still larger supercells) we find the same result as for the $4 \times 4$ supercell, so it seems that hydrogenation of four of the six neighbors, two on each side, is generally sufficient to induce a chemical bond. Tables II and III also show that it is more favorable (in all supercells) to divide the number of hydrogen atoms as symmetrical as possible between the two graphene layers, e.g., the $B B A^{\prime} A^{\prime}$ configuration has lower energy than the $B B B A^{\prime}$ configuration.

In any case it is essential that the $\mathrm{H}$ atoms bind to both sides of the bilayer in order to have an interlayer bond. This means that both sides of the bilayer should be exposed to

TABLE III. Interlayer chemical bond formation in a $3 \times 3$ and $4 \times 4$ supercell. The binding energy per $\mathrm{H}$ atom $\left(E_{b}\right)$ is shown for different numbers of adsorbed hydrogen atoms and different configurations.

\begin{tabular}{lcccc}
\hline \hline Configuration & $E_{b}$ & $\begin{array}{c}3 \times 3 \text { chemical } \\
\text { bond }\end{array}$ & $E_{b}$ & $\begin{array}{c}4 \times 4 \text { chemical } \\
\text { bond }\end{array}$ \\
\hline$B$ & -1.107 & No & -1.225 & No \\
$B B$ & -1.087 & No & -1.131 & No \\
$B A^{\prime}$ & -1.195 & No & -1.296 & No \\
$B B B$ & -1.027 & No & -0.989 & No \\
$B B A^{\prime}$ & -1.169 & No & -1.218 & No \\
$B B B A^{\prime}$ & -1.334 & Yes & -1.042 & No \\
$B B A^{\prime} A^{\prime}$ & -1.466 & Yes & -1.457 & Yes \\
\hline \hline
\end{tabular}

hydrogen since it is known that graphene is very hard to penetrate. ${ }^{24,25}$

Now that we have shown that interlayer chemical bonds are possible, in principle, we will investigate whether they are stable or not. The bond formation is only possible when all $\mathrm{H}$ atoms adsorb on the same sublattice on both sides of the bilayer. We have shown that for a single $\mathrm{H}$ atom there is a difference in binding energy of $0.03 \mathrm{eV}$ in favor of the sublattice that should be hydrogenated. However it has been demonstrated theoretically 22,26 and experimentally ${ }^{27-29}$ that hydrogen atoms tend to adsorb on different sublattices of graphitic surfaces to avoid the presence of unpaired electrons. Boukhvalov and Katsnelson ${ }^{22}$ showed that in this way hydrogen coverage is limited to a maximum of 25 at. \% (on each side) because otherwise first and second neighbors are unavoidably filled and this is energetically unfavorable. We will show, however, that it is possible to reach $50 \%$ hydrogen coverage when the two sides of the bilayer are available for adsorption. For this we will again make use of the $2 \times 2$ supercell and add consecutively more and more $\mathrm{H}$ atoms. There are a lot of different configurations possible, even for a fixed number of adsorbed hydrogen atoms, so we need some guidance to find the most stable ones. We have shown that when the $\mathrm{H}$ atoms adsorb on the same sublattice they will be distributed as symmetrical as possible between the two layers. We can use this as a first guide but, as stated above, there are probably more favorable configurations with the $\mathrm{H}$ atoms distributed over both sublattices. In search of these configurations we make use of earlier theoretical ${ }^{16,22,26}$ and experimental ${ }^{27-29}$ works in which it was found that the formation of two kinds of hydrogen dimers are energetically more favorable after hydrogenation of graphitic surfaces. These dimers are shown in the inset of Fig. 3 together with the most stable trimer configuration, which is a combination of the two dimers, as suggested in Refs. 26 and 28.

As a measure for the stability of the bilayer graphene samples with different concentration of chemisorbed hydrogen atoms we use the formation energy per hydrogen atom. As done by Sofo et al. ${ }^{12}$ we take as reference energies those of graphite and the $\mathrm{H}_{2}$ molecule. The results are shown in Fig. 3.

From our calculations presented in Fig. 3 we can see that for low concentration of $\mathrm{H}$ atoms $(<25$ at. $\%)$ the configurations with hydrogenation of both sublattices is much more stable. This means that no interlayer chemical bonds will be formed for these concentrations. High concentrations of $\mathrm{H}$ atoms $(>25$ at. \%) are not possible for the dimer configurations because the formation energy increases too much, as was already noted in Ref. 22. But if only the B and $A^{\prime}$ sublattices are hydrogenated the formation energy still decreases at high concentration until complete hydrogenation has been reached, i.e., all carbon atoms have four neighbors and are completely saturated (see Fig. 4). The fully hydrogenated bilayer of graphene has negative formation energy as compared to graphite and $\mathrm{H}_{2}$ and is therefore a very stable structure. If we calculate the formation energy with respect to bilayer graphene and atomic hydrogen, as would be typical in an experimental setup, this would result in an almost constant shift of $2.5 \pm 0.1 \mathrm{eV}$ to lower energies which makes all energies given in Fig. 3 negative. This suggests that, in 


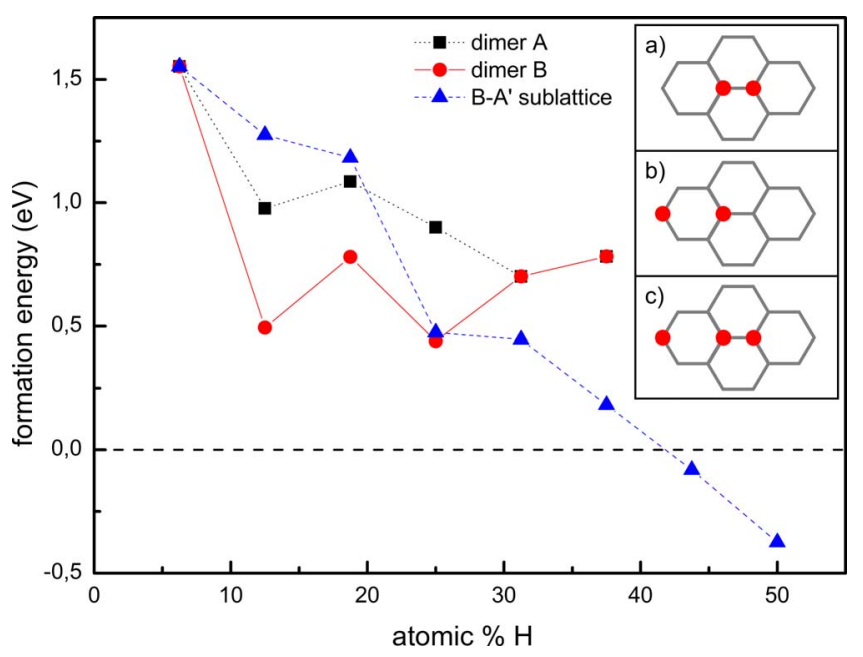

FIG. 3. (Color online) Hydrogenation of $2 \times 2$ supercell of bilayer graphene. The formation energy per hydrogen atom is shown for increasing $\mathrm{H}$ concentration. The three curves show three different ways of distributing the $\mathrm{H}$ atoms: according to dimer configuration $a$ [inset (a)], $b$ [inset (b)], and by placing the $\mathrm{H}$ atoms only on sublattices $\mathrm{B}$ and $\mathrm{A}^{\prime}$. Inset (c) shows the trimer configuration which is a combination of dimer $a$ and $b$. The curves of the two different dimer configurations are the same if the trimer is formed because the trimer is a combination of these two dimers.

principle, all structures could be formed at high enough temperatures so that possible formation barriers can be overcome. ${ }^{16,28}$ Large energy barriers are also expected in going from hydrogenation of both sublattices to hydrogenation of a single sublattice as the hydrogen concentration increases. ${ }^{27}$ One way to avoid these barriers could be to introduce already some interlayer chemical bonds in the bilayer before hydrogenation by, e.g., fs laser excitation ${ }^{30}$ or the introduction of point defects through irradiation. ${ }^{11}$

\section{PROPERTIES OF BILAYER GRAPHANE}

We have shown that the energetically most favorable configuration for hydrogen atoms on a bilayer of graphene ap-

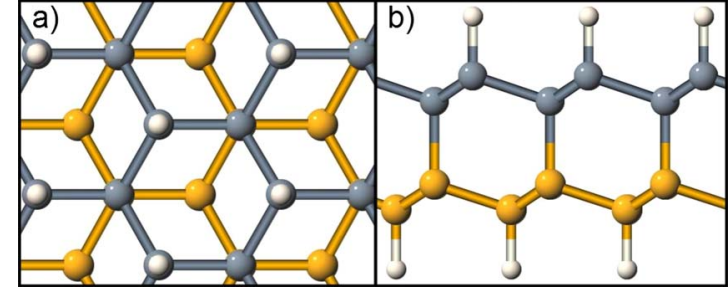

FIG. 4. (Color online) Top (a) and side (b) view of a bilayer of graphane. The carbon atoms of the two layers are given different colors for clarity.

pears when two of the four sublattices (B and $\mathrm{A}^{\prime}$ as indicated in Fig. 1) are fully hydrogenated (see Fig. 4). In this case we could speak of bilayer graphane in analogy with a fully hydrogenated monolayer of graphene. We will now take a look at some properties of this material and compare them to the single layer case and diamond. The results for the geometrical and electronic properties are given in Table IV. First of all we can see that our results for single-layer graphane are in good agreement with earlier studies of this material. ${ }^{12,16}$ The properties of bilayer and single-layer graphane are very similar but there are differences. When we take a look at the geometrical distances and angles, we can see that those of the bilayer are intermediate between those of the single layer and bulk diamond. We also show the corresponding geometrical results for GGA in Table IV because GGA is more accurate in predicting the actual bond lengths. The formation energy of bilayer graphane is larger than that of single-layer graphane but it is closer to the formation energy of graphane than that of diamond. ${ }^{31}$

The electronic properties of both single-layer and bilayer graphane are quite similar (Fig. 5). The band gap of the bilayer is slightly smaller but it is not certain whether this has any physical significance because it is well known that band gaps are not well described in LDA (or GGA). The reason that single and bilayer graphane have similar electronic properties is that the $\mathrm{C}$ atoms in both materials have the same hybridization. So in conclusion we can say that, in contrast to monolayer and bilayer graphene, monolayer and bilayer graphane have very similar electronic properties.

TABLE IV. Properties of single-layer and bilayer graphane: the unit cell length $(a)$, the distances $(d)$ and angles $(\theta)$ between neighboring atoms, the formation energy $\left(E_{F}\right)$, and the band gap at the $\Gamma$ point $(\Delta E)$. Energies are given in $\mathrm{eV}$, distances in $\AA$, and angles in deg.

\begin{tabular}{|c|c|c|c|c|c|c|}
\hline & \multicolumn{2}{|c|}{ Graphane } & \multicolumn{2}{|c|}{ Bilayer graphane } & \multicolumn{2}{|c|}{ Diamond } \\
\hline & LDA & GGA & LDA & GGA & LDA & GGA \\
\hline$a$ & 2.505 & 2.544 & 2.500 & 2.535 & 2.497 & 2.530 \\
\hline$d_{\mathrm{C}-\mathrm{C}}$ & 1.515 & 1.539 & 1.521 & 1.543 & 1.529 & 1.549 \\
\hline$d_{\mathrm{C}-\mathrm{C}^{\prime}}$ & & & 1.545 & 1.562 & 1.529 & 1.549 \\
\hline$\theta_{\mathrm{C}-\mathrm{C}-\mathrm{C}}$ & 111.6 & 111.5 & 110.5 & 110.5 & 109.5 & 109.5 \\
\hline$\theta_{\mathrm{C}-\mathrm{C}-\mathrm{C}^{\prime}}$ & & & 108.4 & 108.4 & 109.5 & 109.5 \\
\hline$\theta_{\mathrm{C}-\mathrm{C}-\mathrm{H}}$ & 107.3 & 107.4 & 108.4 & 108.4 & & \\
\hline$E_{F} /$ atom & -0.183 & -0.104 & -0.125 & -0.028 & -0.010 & 0.122 \\
\hline$\Delta E$ & 3.376 & 3.480 & 3.031 & 3.137 & 5.629 & 5.568 \\
\hline
\end{tabular}



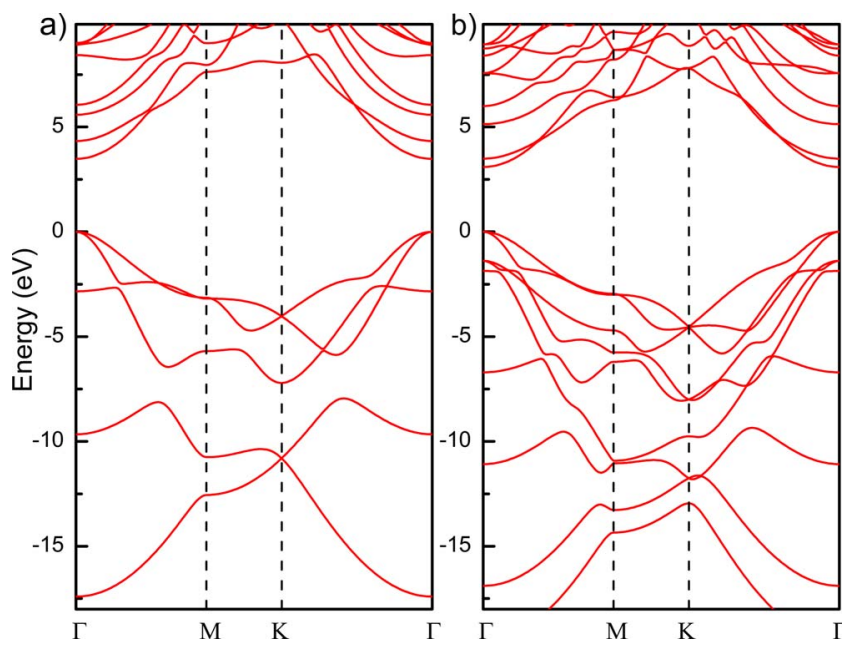

FIG. 5. (Color online) The electronic band structure of (a) monolayer and (b) bilayer graphane. The energies are relative to the Fermi level $\left(E_{F}=0\right)$.

\section{SUMMARY AND CONCLUSIONS}

Previous theoretical calculations showed that hydrogenation of bilayer graphene can only cause a maximum hydro- gen coverage of 25 at. \% when both sublattices (on each side of the bilayer) adsorb $\mathrm{H}$ atoms. ${ }^{22}$ We demonstrated that (1) it is possible to hydrogenate a bilayer of graphene to a limit of 50 at. \% through the adsorption of hydrogen atoms on both sides of the bilayer and on a single sublattice per layer, (2) in this case interlayer chemical bonds are formed which stabilize the structure, and (3) when the hydrogenation reaches its limit of 50 at. \%, a bilayer analog of graphane is formed which has qualitatively similar electronic properties as graphane. Although we have shown that bilayer graphane can be made through the hydrogenation of a bilayer of graphene, this procedure is not suited to produce multilayer $(>2)$ graphane. It is essential for the formation of chemical bonds between two layers that both layers are hydrogenated and this is more difficult when more than two layers are present.

\section{ACKNOWLEDGMENTS}

This work was supported by the Flemish Science Foundation (FWO-Vl), the NOI-BOF of the University of Antwerp, and the Belgian Science Policy (IAP). *ortwin.leenaerts@ua.ac.be

†bart.partoens@ua.ac.be

*francois.peeters@ua.ac.be

${ }^{1}$ K. S. Novoselov, A. K. Geim, S. V. Morozov, D. Jiang, Y. Zhang, S. V. Dubonos, I. V. Grigorieva, and A. A. Firsov, Science 306, 666 (2004).

${ }^{2}$ A. K. Geim and K. S. Novosolov, Nature Mater. 6, 183 (2007).

${ }^{3}$ P. Avouris, Z. Chen, and V. Perebeinos, Nat. Nanotechnol. 2, 605 (2007).

${ }^{4}$ A. K. Geim, Science 324, 1530 (2009).

${ }^{5}$ T. Ohta, A. Bostwick, T. Seyller, K. Horn, and E. Rotenberg, Science 313, 951 (2006).

${ }^{6}$ E. V. Castro, K. S. Novoselov, S. V. Morozov, N. M. R. Peres, J. M. B. Lopes dos Santos, J. Nilsson, F. Guinea, A. K. Geim, and A. H. Castro Neto, Phys. Rev. Lett. 99, 216802 (2007).

${ }^{7}$ S. Patchkovskii, J. S. Tse, S. N. Yurchenko, L. Zhechkov, T. Heine, and G. Seifert, Proc. Natl. Acad. Sci. U.S.A. 102, 10439 (2005).

${ }^{8}$ D. C. Elias, R. R. Nair, T. M. G. Mohiuddin, S. V. Morozov, P. Blake, M. P. Halsall, A. C. Ferrari, D. W. Boukhvalov, A. K. Geim, and K. S. Novosolov, Science 323, 610 (2009).

${ }^{9}$ S. Ryu, M. Y. Han, J. Maultzsch, T. F. Heinz, P. Kim, M. L. Steigerwald, and L. E. Brus, Nano Lett. 8, 4597 (2008).

${ }^{10}$ K. Guérin, J. P. Pinheiro, M. Dubois, Z. Fawal, F. Masin, R. Yazami, and A. Hamwi, Chem. Mater. 16, 1786 (2004).

${ }^{11}$ R. H. Telling, C. P. Ewels, A. A. El-Barbary, and M. I. Heggie, Nature Mater. 2, 333 (2003).

${ }^{12}$ J. O. Sofo, A. S. Chaudhari, and G. D. Barber, Phys. Rev. B 75, 153401 (2007).

${ }^{13}$ X. Gonze, J.-M. Beuken, R. Caracas, F. Detraux, M. Fuchs, G.-M. Rignanese, L. Sindic, M. Verstraete, G. Zerah, F. Jollet,
M. Torrent, A. Roy, M. Mikami, P. Ghosez, J.-Y. Raty, and D. C. Allan, Comput. Mater. Sci. 25, 478 (2002).

${ }^{14}$ M. Torrent, F. Jollet, F. Bottin, G. Zerah, and X. Gonze, Comput. Mater. Sci. 42, 337 (2008).

${ }^{15}$ H. J. Monkhorst and J. D. Pack, Phys. Rev. B 13, 5188 (1976).

${ }^{16}$ D. W. Boukhvalov, M. I. Katsnelson, and A. I. Lichtenstein, Phys. Rev. B 77, 035427 (2008).

${ }^{17}$ M. Hasegawa and K. Nishidate, Phys. Rev. B 70, 205431 (2004).

${ }^{18}$ Y. Baskin and L. Meyer, Phys. Rev. 100, 544 (1955).

${ }^{19}$ R. Zacharia, H. Ulbricht, and T. Hertel, Phys. Rev. B 69, 155406 (2004).

${ }^{20}$ Zahlenwerte und Funktionen aus Naturwissenchaften und Tech$n i k$, edited by O. Madelung and M. Schulz, Landolt-Börnstein, New Series, Group III, Vol. 22, Pt. A (Springer-Verlag, Berlin, 1987).

${ }^{21}$ G. Zilibotti, M. C. Righi, and M. Ferrario, Phys. Rev. B 79, 075420 (2009).

${ }^{22}$ D. W. Boukhvalov and M. I. Katsnelson, Phys. Rev. B 78, 085413 (2008)

${ }^{23}$ Z. Luo, T. Yu, K.-j. Kim, Z. Ni, Y. You, S. Lim, Z. Shen, S. Wang, and J. Lin, ACS Nano 3, 1781 (2009).

${ }^{24}$ J. S. Bunch, S. S. Verbridge, J. S. Alden, A. M. van der Zande, J. M. Parpia, H. G. Craighead, and P. L. McEuen, Nano Lett. 8, 2458 (2008).

${ }^{25}$ O. Leenaerts, B. Partoens, and F. M. Peeters, Appl. Phys. Lett. 93, 193107 (2008).

${ }^{26}$ S. Casolo, O. M. Løvvik, R. Martinazzo, and G. F. Tantardini, J. Chem. Phys. 130, 054704 (2009).

${ }^{27}$ L. Hornekær, Ž. Šljivančanin, W. Xu, R. Otero, E. Rauls, I. Stensgaard, E. Lægsgaard, B. Hammer, and F. Besenbacher, 
Phys. Rev. Lett. 96, 156104 (2006).

${ }^{28}$ L. Hornekær, E. Rauls, W. Xu, Ž. Šljivančanin, R. Otero, I. Stensgaard, E. Lægsgaard, B. Hammer, and F. Besenbacher, Phys. Rev. Lett. 97, 186102 (2006).

${ }^{29}$ N. P. Guisinger, G. M. Rutter, J. N. Crain, P. N. First, and J. A. Stroscio, Nano Lett. 9, 1462 (2009).

${ }^{30}$ J. Kanasaki, E. Inami, K. Tanimura, H. Ohnishi, and K. Nasu, Phys. Rev. Lett. 102, 087402 (2009).

${ }^{31}$ Note that the formation energy for diamond is negative in the LDA case although it is known from experiment that graphite is more stable than diamond (Ref. 32). This is a consequence of the underestimation of the interlayer vdW interaction energy in graphite as can be seen from Table I. If we include the vdW interaction energy (we realize this artificially by adding the dif- ference between the experimental and LDA values for the interlayer binding energy (see Table I) to the formation energy), the formation energy of diamond is $25 \mathrm{meV}$ which is close to the experimental result of $\sim 0.03 \mathrm{eV}$ (Ref. 32). In agreement with earlier GGA calculations (Ref. 33) we find that GGA overestimates the formation energy of diamond. One can observe also a substantial difference between LDA and GGA for the formation energies of single and bilayer graphane. This is probably due to the overestimation of the C-H bond energy in LDA (Ref. 16).

${ }^{32}$ S. Fahy, X. W. Wang, and S. G. Louie, Phys. Rev. B 42, 3503 (1990).

${ }^{33}$ A. Janotti, S.-H. Wei, and D. J. Singh, Phys. Rev. B 64, 174107 (2001). 